\title{
Network Externalities in a Dynamic Monopoly
}

\author{
Luca Lambertini* - Raimondello Orsini** \\ Dipartimento di Scienze Economiche \\ Università di Bologna \\ Strada Maggiore 45, 40125 Bologna, Italy \\ phone: +39-051-2092600, fax: +39-051-2092664 \\ *lamberti@spbo.unibo.it, **orsini@spbo.unibo.it
}

November 18,2002

\begin{abstract}
We reconsider the role of network externalities in a dynamic spatial monopoly where the firm must invest in order to accumulate capacity, while consumers may have either linear or quadratic preferences. We (i) characterise saddle point equilibria, (ii) prove that the extent of market coverage is increasing in the network effect and (iii) unlike the existing static literature on the same problem, the monopolist may not make introductory price offers. Then, we briefly deal with the socially optimal solution, showing that, in general, a planner would serve more consumers than a profit-seeking monopolist.
\end{abstract}

Keywords: network externality, spatial monopoly, capital accumulation, optimal control

JEL Classification: D62, D92, L12 


\section{Introduction}

There exist several markets for goods whose consumption involves relevant network effects, such that the utility that a consumer derives from the purchase of a good or service is increasing in the number of other consumers doing the same. The software and telecommunications industries and, more generally, the markets for information goods, are examples of sectors where such externalities operate.

Intuitively, joining the network becomes increasingly attractive to the generic consumer as the size of the network becomes larger over time. Notwithstanding the fact that this phenomenon is intrinsically dynamic, the existing literature treats it as a static one (see Economides and Encaoula, 1996; Shy, 2000). Ever since Rohlfs (1974), the basic questions addressed in this literature are: how should a monopolist price a good that is subject to network externalities? In particular, should the monopolist set a low introductory price to attract a critical mass of adopters? To illustrate these issues, we briefly summarise the basic model as in Shy (1998, pp. 256-9). A unit mass of consumers is uniformly distributed over $[0,1]$, in decreasing order w.r.t. their willingness to enter the network. The value of joining the network is increasing in the network size. Therefore, the overall willingness to pay for the good or service of a consumer at $m \in[0,1]$ is $w=y(1-m)$, where $y$ is the size of the network, i.e., the market demand for that good. The consumer's net surplus is $U=y(1-m)-p$, where $p$ is the market price. In order to determine demand $y$, the firm identifies the marginal consumer in $m=y$ and sets $p=y(1-y)$. Now observe that, being the overall willingness to pay of the marginal consumer, $\bar{w}=y(1-y)$, concave in $y$, for any given price $p<1 / 4$ there are two economically admissible sizes of the network, as it appears from figure 1.

This model is usually considered as blackboxing an underlying dynamic process whereby consumers enter sequentially the network, starting from the left boundary of the unit interval. The adjustment is driven by the assumption that $y$ increases whenever $p<y(1-y)$, and conversely. Hence, as soon as $y_{1}$ consumers buy at price $\bar{p}$, the market immediately jumps into $\left\{y_{2}, \bar{p}\right\}$. This amounts to saying that $\left\{y_{1}, \bar{p}\right\}$ is unstable. Nevertheless, its economic interest lies in the fact that $y_{1}$ is the so-called critical mass, ensuring that marketing the product is going to be successful. 
Figure 1: Equilibrium network sizes

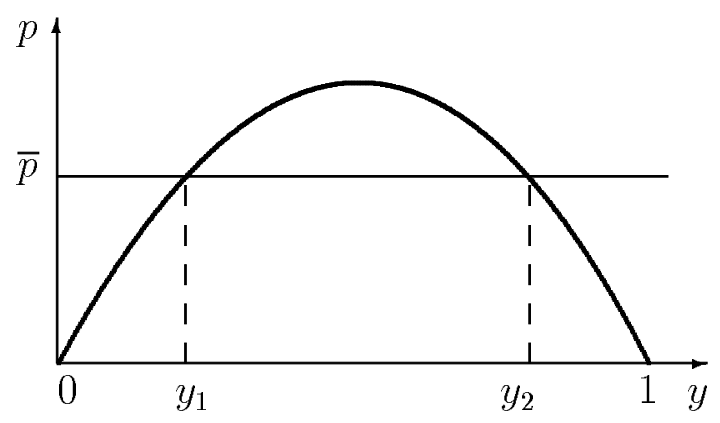

We propose a properly dynamic reformulation of this problem as an optimal control model that can be formalised in two slightly different ways, showing that the issue of the critical mass disappears in both cases. To this purpose, we use a spatial model à la Hotelling (1929), with either linear or quadratic disutility associated with the distance between firm and consumers. In the first case, there exists a unique steady state, which is both economically admissible and stable in the saddle sense. In the second, there indeed exist two steady states, but one is both unstable and economically meaningless. However, the model preserves an intuitive feature of network economics, namely, the monopoly output is everywhere non-decreasing in the extent of the network effect. Therefore, the need for regulation becomes less urgent as the network externality grows larger (see Cabral, Salant and Woroch, 1999; Lambertini and Orsini, 2001).

The basic model is in section 2. Section 3 contains the analysis of the linear transportation cost setting, while the alternative setting with quadratic disutility is investigated in section 4 . The issues of introductory price offers and critical mass are discussed in section 5. The behaviour of a benevolent planner maximising welfare is investigated in section 6 . Concluding remarks are in section 7 .

\section{The setup}

Consider a monopoly market over an infinite (continuous) time horizon, $t \in[0, \infty)$. As in Hotelling (1929), consumer tastes are uniformly distributed 
with density 1 over $[0,1]$. The maximum intrinsic satisfaction from the consumption of one unit of the good is $s$ (which is assumed to be nil in the static model sketched above). The generic consumer at $m \in[0,1]$ buys one unit of the good iff:

$$
U=s-f(m-x(t))+\alpha y(t)-p(t) \geq 0
$$

where $p(t)$ and $x(t)$ are the price and the location chosen by the monopolist at time $t$; the function $f(m-x(t))$ measures the disutility caused by consuming a product not coinciding with own taste, and we assume that it is strictly increasing in $m-x(t) ; \alpha y(t)$ is the network externality which is assumed to be linear in the market demand $y(t)$. When inequality $(1)$ is reversed, the consumer located at $m$ does not buy and his utility is $U=0$.

Production involves a constant marginal cost $c<s$. In order to produce the output $y(t)$ demanded by the market, the firm must build up capacity $k(t)=y(t)$. This involves an investment process accumulating capacity according to the following dynamics à la Solow (1956) or Nerlove and Arrow (1962):

$$
\dot{k}=\dot{y}=I(t)-\delta y(t)
$$

where $I(t)$ is the instantaneous investment and $\delta \in[0,1]$ is a constant depreciation rate. The instantaneous cost involved by investing $I(t)$ is $C(I(t))=b[I(t)]^{2}$. Hence, instantaneous monopoly profits are:

$$
\pi_{M}(t)=[p(t)-c] y(t)-b[I(t)]^{2}
$$

and, given a constant discount rate $\rho$, the monopolist must choose $y(t), I(t)$ and $x(t)$ so as to maximise:

$$
\begin{aligned}
\Pi_{M} \equiv \int_{0}^{\infty}\left\{[p(t)-c] y(t)-b[I(t)]^{2}\right\} e^{-\rho t} d t \\
\text { s.t. }: \dot{y}=I(t)-\delta y(t) .
\end{aligned}
$$

It can be easily shown that the monopolist cannot do any better than locating at $1 / 2$ (see, e.g., Bonanno, 1987). Therefore, in the remainder, we shall set $x=1 / 2$.

\section{$3 \quad$ Linear disutility}

Suppose $f(m-x(t)) \equiv v|m-x(t)|, v>0$. If so, the price that drives to zero the two marginal consumers symmetrically located to the left and to the 
right of $x=1 / 2$ is:

$$
p_{M}=s-\frac{y(t)}{2}(v-2 \alpha)
$$

and the relevant Hamiltonian is the following:

$$
\begin{aligned}
\mathcal{H}= & e^{-\rho t}\left\{y(t)\left[s-\frac{y(t)}{2}(v-2 \alpha)-c\right]\right. \\
& \left.-b[I(t)]^{2}+\lambda[I(t)-\delta y(t)]\right\},
\end{aligned}
$$

where $\lambda(t)=\mu(t) e^{\rho t}, \mu(t)$ being the co-state variable associated to $y(t)$. The initial condition is $y(0) \in[0,1)$, while the transversality condition is $\lim _{t \rightarrow \infty} \lambda(t) y(t)=0$. The first order conditions (FOCs) of the monopolist are (henceforth we omit the indication of time and discounting for brevity):

$$
\begin{aligned}
\frac{\partial \mathcal{H}}{\partial I} & =-2 b I+\lambda=0 \\
-\frac{\partial \mathcal{H}}{\partial y} & =\dot{\lambda}-\rho \lambda \Rightarrow \\
\dot{\lambda} & =y(v-2 \alpha)-s+c+\lambda(\rho+\delta) .
\end{aligned}
$$

From $(7)$, one obtains $\lambda=2 b I$ and $I=\lambda /(2 b)$. Hence:

$$
\dot{I}=\frac{y(v-2 \alpha)-s+c+2 b I(\rho+\delta)}{2 b}=0
$$

in $I=[s-c-y(v-2 \alpha)] /[2 b(\rho+\delta)]$ which can be plugged into $\dot{k}=0$ to yield the optimal monopoly output in steady state:

$$
\begin{aligned}
& y_{M}=\frac{s-c}{v-2 \alpha+2 b \delta(\rho+\delta)}>0 \\
& \forall \alpha<\frac{v+2 b \delta(\rho+\delta)}{2} .
\end{aligned}
$$

If $s \in(c, v+c+2 b \delta(\rho+\delta))$,

$$
y_{M} \in[0,1] \forall \alpha \in\left[0, \frac{v+c-s+2 b \delta(\rho+\delta)}{2}\right] ;
$$

with $I_{M}=\delta y_{M}$. In this parameter range, $y_{M}$ and $I_{M}$ are monotonically increasing in $\alpha$ and it can be easily checked that $\left\{I_{M}, y_{M}\right\}$ is a saddle point. 
Then, $y_{M}=1$ for all $\alpha \geq v-s+c+2 b \delta(\rho+\delta)$. If $s>v+c+2 b \delta(\rho+\delta)$, all consumers in the market are served irrespective of $\alpha$.

Using (5) and (10), one can write the expression of the steady state price, to verify that

$$
\frac{\partial p_{M}}{\partial \alpha}=\frac{2 b \delta(\rho+\delta)(s-c)}{[v-2 \alpha+2 b \delta(\rho+\delta)]^{2}}>0 .
$$

\section{Quadratic disutility}

Now assume $f(m-x(t)) \equiv v[m-x(t)]^{2}$. The price driving to zero the two marginal consumers symmetrically located to the left and to the right of $x=1 / 2$ is:

$$
p_{M}=s-\frac{v[y(t)]^{2}}{4}+\alpha y(t)
$$

and the Hamiltonian is:

$$
\begin{aligned}
\mathcal{H}= & e^{-\rho t}\left\{y(t)\left[s-\frac{v[y(t)]^{2}}{4}+\alpha y(t)-c\right]\right. \\
& \left.-b[I(t)]^{2}+\lambda[I(t)-\delta y(t)]\right\},
\end{aligned}
$$

where $\lambda(t)=\mu(t) e^{\rho t}$. Initial and transversality conditions are as in section 3 . The FOCs of the monopolist are (again, henceforth we omit the indication of time and discounting):

$$
\begin{aligned}
\frac{\partial \mathcal{H}}{\partial I} & =-2 b I+\lambda=0 \\
-\frac{\partial \mathcal{H}}{\partial y} & =\dot{\lambda}-\rho \lambda \Rightarrow \\
\dot{\lambda} & =\frac{3 v y^{2}}{4}-2 \alpha y-s+c+\lambda(\rho+\delta) .
\end{aligned}
$$

From (15), one obtains $\lambda=2 b I$ and $\dot{I}=\dot{\lambda} /(2 b)$. Imposing $\dot{I}=0$ yields

$$
I=\frac{4(s-c)+y(8 \alpha-3 v y)}{8 b}
$$

which can be plugged into $\dot{y}=0$ to obtain:

$$
y=\frac{z \pm \sqrt{z^{2}+3 v(s-c)}}{3 v}
$$


where $z \equiv \alpha-b \delta(\rho+\delta)$. Since $s>c$, the smaller root in (18) is negative. Hence, there is only one economically admissible solution yielding the steady state network size $y_{M}$, which is always positive and must be at most equal to one. To this regard, a sufficient condition for the larger root in (18) to be at least one is $\alpha \geq 3 v+b \delta(\rho+\delta)$. Therefore, in this range, $y_{M}=1$. If instead $\alpha<3 v+b \delta(\rho+\delta)$, the larger root belongs to $[0,1]$ for all

$$
\begin{aligned}
& \alpha \leq \widetilde{\alpha} \equiv \frac{3 v-4(s-c)+8 b \delta(\rho+\delta)}{8} \\
& \widetilde{\alpha}>0 \forall s \in\left(c, \frac{4 c+3 v+8 b \delta(\rho+\delta)}{4}\right)
\end{aligned}
$$

which entails that, for sufficiently high values of $s$, the market is fully covered irrespective of $\alpha$. The steady state investment is $I_{M}=\delta y_{M}$. The analysis of the Jacobian matrix of the problem shows that the pair $\left\{I_{M}, y_{M}\right\}$ is a saddle point. It can also be ascertained that $\partial p_{M} / \partial \alpha>0$ in the range where $y_{M} \in[0,1]$. The details of this result are omitted for brevity.

The discussion carried so far establishes:

Proposition 1 Irrespective of whether consumer preferences contain a linear or quadratic disutility component, there exists a unique steady state point, which is a saddle. The optimal output and price are both non-decreasing in the network effect.

\section{$5 \quad$ Introductory offers and critical mass}

Now, using (5) and (13), we examine the possibility of introductory price offers by the monopolist, i.e., the sign of $\dot{p}$ before reaching the steady state. In the linear case:

$$
\dot{p}>0 \forall \alpha>v / 2
$$

entailing that the monopolist makes an introductory offer to encourage consumers to join the network only if the network effect is sufficiently heavy. Since condition (21) depends only on exogenous parameters, the evolution of price is monotone over time.

As to the quadratic case, the evolution of price establishes that

$$
\dot{p}>0 \forall \alpha>v y / 2 \text {. }
$$


Here, the willingness to make an introductory offer depends also on the current network size. Hence, the price dynamics can be non-monotone over time. As the introductory offer is part of the monopolist's initial behaviour, it is affected by the initial condition $y(0)$. The closer $y(0)$ is to zero, the easier is for $\dot{p}>0$ to hold.

Proposition 2 Ceteris paribus, the likelyhood of an initial price offer is higher with quadratic disutility than with linear disutility.

Note that, in general, if $\alpha=0, \dot{p} \propto-\dot{y}<0$ always. That is, network externalities being absent, price would monotonically decrease over time.

Concerning the issue of the critical mass, we can briefly deal with the linear model, where equations (5) and (10) clearly imply that, the solution being unique, the issue of a critical mass does not arise. As to the quadratic case, from (13) and (18) we know that there are two steady state points. However, for $s>c$, the smaller solution is not only unstable (as it can be checked from the Jacobian matrix), but also negative, hence economically meaningless. Consequently, there exists no critical mass in the quadratic formulation either. This deserves an additional remark, as there can be goods or services generating network effects, but without any intrinsic utility (i.e., $s=0$ ). This can be the case, e.g., for telephones and other communication services, while for the vast majority of network goods $s>c$ appears to be a sensible assumption. E.g., being the only owner of a computer or a CD player, in addition to a network effect, yields surely an intrinsic satisfaction, implying a willingness to pay surely higher than unit production cost. ${ }^{1}$ If indeed $s \in[0, c)$, the smaller solution in (18) is positive as long as it is real. However, it remains unstable, and the dynamic properties of the system drive the monopolist to the saddle point. Only in this case there arises a critical threshold for market demand.

\section{The social optimum}

The objective of a benevolent social planner is to maximise

$$
\begin{gathered}
\int_{0}^{\infty} S W(t) e^{-\rho t} d t \\
\text { s.t. }: \dot{y}=I(t)-\delta y(t)
\end{gathered}
$$

\footnotetext{
${ }^{1}$ Note that $s$ is the intrinsic satisfaction of the happiest consumer located at $1 / 2$ and surely buying at $t=0$ for any initial condition, i.e., the one for whom the disutility component is nil.
} 
where instantaneous social welfare $S W(t)$ is defined as the sum of profits, defined in (3), and consumer surplus $C S(t)$, which is computed as follows:

$$
C S=2 \int_{1 / 2}^{m}\left[s+\alpha y-p-f\left(g-\frac{1}{2}\right)\right] d g .
$$

For brevity, we omit computational details on the Hamiltonian of the planner. If the disutility is linear, i.e., $f(\cdot)=v|g-1 / 2|$, the socially optimal steady state output is

$$
y_{S}=\min \left\{\frac{2(s-c)}{v-4 \alpha+4 b \delta(\rho+\delta)}, 1\right\} .
$$

If instead the disutility is quadratic, the steady state solution is:

$$
y_{S}=\min \left\{\frac{4 z+2 \sqrt{4 z^{2}+v(s-c)}}{v}, 1\right\} \text {. }
$$

In both cases, $y_{S}>y_{M}$ in the parameter set where $y_{M} \in[0,1)$. Moreover, it is easily shown that the parameter region where the planner fully covers the market is wider than the region where the monopolist behaves likewise.

\section{Conclusions}

We have assessed network effects in a dynamic spatial monopoly where the firm must invest in order to accumulate capacity, while consumers may have either linear or quadratic preferences. For both specifications of consumer utility, we have shown that the model produces a unique saddle point equilibrium which is economically meaningful, whereby we can conclude that the issue of the critical mass, widely discussed in the static literature, generally does not arise in our dynamic setting. We have also proved that, contrary to the conclusions reached by the existing static literature on the same problem, the monopolist may not necessarily choose to make introductory price offers to attract customers. To complete the picture, we have briefly investigated the socially optimal solution, showing that, in general, a planner would serve more consumers than a profit-seeking monopolist. 


\section{References}

[1] Bonanno, G. (1987). Location choice, product proliferation and entry deterrence. Review of Economic Studies, 54, 37-46.

[2] Cabral, L., D. Salant and G. Woroch (1999). Monopoly pricing with network externalities. International Journal of Industrial Organization, 17, 199-214.

[3] Economides, N. and D. Encaoua (1996, Eds.). Special issue on network economics: business conduct and market structure. International Journal of Industrial Organization, 14.

[4] Hotelling, H. (1929). Stability in competition. Economic Journal, 39, 41-57.

[5] Lambertini, L. and R. Orsini (2001). Network externalities and the overprovision of quality by a monopolist. Southern Economic Journal, 67, 969-982.

[6] Nerlove, M. and K.J. Arrow (1962). Optimal advertising policy under dynamic conditions. Economica, 29, 129-142.

[7] Rohlfs, J. (1974). A theory of interdependent demand for a communications service. Bell Journal of Economics, 5, 16-37.

[8] Shy, O. (1998). Industrial Organization. Theory and Applications. Cambridge, MA, MIT Press.

[9] Shy, O. (2000). The Economics of Network Industries. Cambridge, Cambridge University Press.

[10] Solow, R. (1956). A contribution to the theory of economic growth. Quarterly Journal of Economics, 70, 65-94. 